\title{
Five-year incidence of type 2 diabetes mellitus among cardiovascular disease-free Greek adults: Findings from the ATTICA study
}

\author{
Demosthenes B \\ Panagiotakos' \\ Christos Pitsavos ${ }^{2}$ \\ Yannis Skoumas ${ }^{2}$ \\ Yannis Lentzas ${ }^{2}$ \\ Christodoulos Stefanadis ${ }^{2}$ \\ 'Department of Nutrition Science- \\ Dietetics, Harokopio University, \\ Athens, Greece; ${ }^{2}$ First Cardiology \\ Clinic, School of Medicine, University \\ of Athens, Athens, Greece
}

\begin{abstract}
Objective: We evaluated the 5-year incidence of diabetes in an adult population from Greece.

Research design and methods: 3042 individuals ( $>18$ years), free of cardiovascular disease, participated in the baseline examination (during 2001-2002). Of this sample, 1012 men and 1035 women were found alive at the time of follow-up, while $32(2.1 \%)$ men and $22(1.4 \%)$ women died during this period. The rest were lost to follow-up. Incidence of type 2 diabetes mellitus was evaluated in 1806 participants who did not have diabetes at baseline.

Results: The age-adjusted 5-year incidence of diabetes was 5.5\% (men, 5.8\%; women, 5.2\%). A linear trend was observed between diabetes incidence and age (5.6\% increases in incidence per 1 -year difference in age, $\mathrm{p}<0.001)$. Multiple logistic regression analysis revealed that age (OR per $1 \mathrm{yr}=1.04,95 \%$ CI $1.02-1.06$ ), waist (OR per $1 \mathrm{~cm}=1.02,95 \%$ CI $1.01-1.003$ ), physical activity $(\mathrm{OR}=0.62,95 \% \mathrm{CI} 0.35-1.02)$ and family history of diabetes $(\mathrm{OR}=2.65,95 \%$ CI 1.58-4.53), as well as fasting glucose levels (OR per $1 \mathrm{mg} / \mathrm{dl}=1.05,95 \%$ CI 1.03-1.07), were the most significant baseline predictors for diabetes, after adjusting for various potential confounders. Additionally, presence of metabolic syndrome at baseline evaluation 2.95-fold the risk of diabetes (95\% CI 1.89-4.61), and showed better classification ability than the model that contained the components of the syndrome (ie, correct classification rate: $94.5 \%$ vs. $92.3 \%$ ).

Conclusion: Our findings show that a 5.5\% incidence rate of diabetes within a 5-year period, which suggests that the prevalence of this disorder in Greece is rising. Aging, heredity, and metabolic syndrome were the most significant determinants of diabetes.
\end{abstract}

Keywords: diabetes, incidence, metabolic syndrome

An international diabetes federation that includes more than 150 countries, estimates that the prevalence of type 2 diabetes mellitus is raising persistently and affects about $6 \%$ of people aged 20-79 years; most of them live in developing countries (Mayor 2006). It is also of interest that the forecasts are at alarming rates, while a considerable proportion of diabetic people are unaware of their condition (King et al 1998). Diabetes mellitus is a major contributory cause for heart attacks, blindness, strokes, kidney failure and impotence (CDC 2005). Fortunately, there are is convincing evidence from randomized clinical trials and prospective epidemiologic studies that those lifestyle modifications, including healthy diet, smoking cessation, exercise, and education can minimize the burden of the disease (vanDam et al 2002; Panagiotakos et al 2005d; Patja et al 2005; Tuomilehto and Wareham 2006).

Despite the clinical importance of diabetes, data about the incidence of the disease in developing countries and particularly in low cardiovascular risk populations are lacking. In Greece, results from previous observational studies show that the self-reported 
diabetes was increased from $2.4 \%$ to $3.1 \%$, between 1974 and 1990 (Katsilambros et al 1993), while the prevalence of type 2 diabetes in the ATTICA's study sample in 2001, was $7.6 \%$ in men and 5.9\% in women (Panagiotakos et al 2005a). These figures are in accordance with the incidence rates of diabetes worldwide. Nevertheless, the incidence of type 2 diabetes and the significance of factors affecting the development of the disease in the Greek population have never been examined. Thus, in this work we sought to examine the 5-year incidence of diabetes, in a sample of cardiovascular diseasefree adults from the Attica region, in Greece (ie, the ATTICA study; Pitsavos et al 2003), and we have also evaluated the effect of several clinical, biological, and lifestyle factors on the development of the disease.

\section{Methods}

\section{Participants}

The ATTICA epidemiological study (Pitsavos et al 2003; Panagiotakos et al 2005a) is a health and nutrition survey that has been carried out in the province of Attica region (including 78\% urban and 22\% rural areas), during 20012002. People with cardiovascular disease or people living in institutions were excluded from the sampling. According to the study's design, 4056 inhabitants from the above area were randomly selected to enroll; of them, 3042 agreed to participate ( 1514 were men aged $46 \pm 13$ years and 1528 were women aged $45 \pm 13$ years). All participants interviewed by trained personnel (cardiologists, dieticians, and nurses) who used a standard questionnaire.

\section{Baseline measurements}

The baseline evaluation included: demographic characteristics (age, sex, and years of school as proxy of social status), detailed information regarding personal and family history of hypertension, hypercholesterolemia, diabetes, dietary and other lifestyle habits, such as smoking status, and habitual/leisure time physical activity. In particular, the evaluation of the nutritional habits was based on a validated semi-quantitative food-frequency questionnaire (Katsouyanni et al 1997). All participants were asked to report the average intake (per week or day) of several food items that they consumed (during the last 12 months). Alcohol consumption was measured in wine glasses $(100 \mathrm{ml})$ and quantified by ethanol intake (grams per drink). One wine glass was considered to have an equivalent of $12 \%$ ethanol concentration. Composite scores were used to describe overall diet, which are necessary for the evaluation of epidemiological associations. Thus, a special Mediterranean diet score was used (range 0-55), based on the rationale of the Mediterranean dietary pyramid (Panagiotakos et al 2006). Smokers were defined as those who were smoking at least one cigarette per day during the past year or had recently stopped smoking (during a year); the rest of the participants were defined as nonsmokers. For the ascertainment of physical activity status the International Physical Activity Questionnaire was used (IPAQ 2006), as an index of weekly energy expenditure using frequency (times per week), duration (in minutes per time), and intensity of sports or other habits related to physical activity (in expended calories per time). According to the IPAQ, participants were classified as: (a) minimally active or inactive, (b) moderately active achieving of at least $600 \mathrm{MET}$-min/week, and (c) highly active when achieving a minimum of at least 1500 MET-minutes/week, or 7 or more days of any combination of walking, moderate-intensity or vigorous intensity activity achieving a minimum of at least 3000 MET-minutes/week. Body mass index was measured as weight (in kilograms) divided by standing height (in meters squared). Obesity was defined as body mass index $>29.9 \mathrm{~kg} / \mathrm{m}^{2}$. Waist circumference (in $\mathrm{cm}$ ) was also measured using standard procedures. Arterial blood pressure was measured at the end of the physical examination with subject in sitting position. All participants were at least 30 minutes at rest. Participants whose average of three consecutive measurements of blood pressure levels were greater or equal to $140 \mathrm{and} /$ or $90 \mathrm{mmHg}$ or reported that they were under antihypertensive medication were classified as having hypertension. Blood samples were collected from the antecubital vein between 8 to $10 \mathrm{AM}$, in a sitting position after 12 hours of fasting and alcohol abstinence. Total-, high-density lipoprotein (HDL), cholesterols, blood glucose, and triglycerides were also measured in all participants, using colorimetric enzymic method in a Technicon automatic analyzer RA-1000 (DateBehring Marburg GmbH, Marburg, Germany). Low-density lipoprotein-cholesterol was calculated using the Friedwald method. The intra and inter-assay coefficients of variation of cholesterol levels did not exceed $4 \%$, triglycerides $4 \%$, and glucose 4\%. Hypercholesterolemia was defined as total cholesterol levels greater than $200 \mathrm{mg} / \mathrm{dl}$ or the use of lipid-lowering agents. High-sensitivity C-reactive protein levels (as markers of low-grade systemic inflammation) were assayed by particle-enhanced immunonephelometry (N Latex; Dade-Behring Marburg $\mathrm{GmbH}$ ) with a range from 0.175 to $1100 \mathrm{mg} / \mathrm{dl}$ and 0.75 to $1000 \mathrm{mg} / \mathrm{dl}$, respectively. The intra-assay and inter-assay coefficient of variation was $<5 \%$. 


\section{Follow-up}

From June to August 2006, the ATTICA study investigators performed the 5-year follow-up. Of the 3042 individuals that participated in the baseline examination, 1012 men and 1035 women were found alive at the time of the follow-up, while $32(2.1 \%)$ men and $22(1.4 \%)$ women died (cardiovascular disease [32], cancer [14], and other causes [8]) during the 5 -year period ( $69 \%$ follow-up participation rate). Of the participants that did not participate in the re-examination, $25 \%$ were not found because of missing or wrong addresses and telephone numbers and $5 \%$ declined to be re-examined. No significant differences were observed between those who were lost to follow-up and the rest of the participants regarding sex $(p=0.99)$, age $(p=0.78)$, education level in years of school $(p=0.67)$, presence of hypertension $(p=0.12)$, diabetes $(p=0.27)$, and hypercholesterolemia $(p=0.12)$. The re-examination was based on telephone interviews ( $80 \%$ of the participants) and on face-to-face interviews when telephone number was not available. No differences were observed between those contacted by telephone and those participated in the face-to-face interviews, regarding age $(p=0.77)$, sex $(p=0.89)$, and place of living $(p=0.98)$. The re-examination included information about: (a) vital status (death from any cause), or development of coronary heart disease or stroke, (b) development of hypertension, hypercholesterolemia and type 2 diabetes (ie, fasting blood glucose levels $>125 \mathrm{mg} / \mathrm{dl}$ or use of antidiabetic medication), among people who did not have these disorders at baseline, as well as management of these conditions, (c) assessment of body weight, and (d) lifestyle habits, including physical activity, smoking status, and intake of various foods. For the present analysis people who have been defined as diabetic at baseline examination were excluded. Thus, taking into account those who were lost to follow-up, data from 1806 participants were analyzed in this work.

\section{Statistical analysis}

Continuous variables are presented as mean values \pm standard deviation. Categorical variables are presented as absolute and relative frequencies. Associations between categorical variables were tested using the chi-squared test. Comparisons of mean values of normally distributed variables between those who developed diabetes and the rest of the participants were performed using the Student's t-test. Since the exact time to event (ie, development diabetes) was not known, the relative risks of developing the disease during the 5-year follow-up period were estimated using the odds ratios and their corresponding 95\% confidence intervals through stepwise multiple logistic regression analysis. We used 5\% as the cut-off for the probability of entering a variable in the model and $10 \%$ as the cut-off for the probability of removing a variable from the model. The probabilities were calculated using the Wald test. Interactions between sex and other covariates were tested in all steps, and when they were significant, remained in the final model. Deviance residuals were used to evaluate model's goodness-of-fit and -2loglikelihood of the initial and final model were also calculated. All reported p-values are based on two-sided tests and compared to a significance level of 5\%. SPSS version 14 (Statistical Package for Social Sciences, SPSS Inc, Chicago, IL, USA) software was used for all the statistical calculations.

\section{Results}

During the period 2001-2006, the crude incidence of diabetes was 58 per 1000 men and 53 per 1000 women $(p=0.64)$. Therefore, it could be speculated that the annual incidence rate is $1.16 \%$ in men and $1.06 \%$ in women. The men-towomen incidence rate ratio was approximately 1-to-1, in almost all age groups. However, in people between 65-75 years old men were 1.5-times more likely to develop diabetes compared with women of same age, while in older than 75 years participants, women were 1.56 -times more likely to develop the disease compared with men (Table 1). As we can see in Table 1, a strong linear trend of diabetes incidence was observed across all age groups, in both sexes. In particular, it was found that the crude risk of diabetes increased by $5.6 \%$ per 1-year difference in age (95\% CI 4\%-7.2\%).

The distribution of the baseline demographic parameters and cardiovascular risk factors levels, into those who were diagnosed as diabetic at follow-up and the rest of the participants is presented in Table 2. Unadjusted analysis revealed several significant relationships. For example, people who developed diabetes were away from the traditional Mediterranean diet (ie, lower diet score), were more likely to have hypertension, obesity, and hypercholesterolemia at baseline, and had higher body mass index and waist circumference. In addition, people who developed diabetes were less educated; the incidence of diabetes in people with low education (ie, $<6$ years of school) was $11.0 \%$, while the incidence among people with high education (ie, $>12$ years of school) was $3.8 \%(\mathrm{p}<0.001)$.

Although, several differences were observed between those who developed diabetes and those who did not regarding their baseline characteristics (Table 2), residual confounding may exist. Thus, multi-adjusted analysis was then applied and revealed that age, waist circumference, physical inactivity and family history of diabetes, were the baseline factors that significantly predicted the development of diabetes (see final 
Table I Five-year incidence of type 2 diabetes mellitus in men and women by age group.

\begin{tabular}{clll}
\hline & Men & \multicolumn{2}{l}{ Women } \\
\hline \# People participated in the follow-up & 880 & 926 & \\
Age at baseline & $\begin{array}{l}\text { 5-year incidence } \\
\text { of diabetes }\end{array}$ & $\begin{array}{l}\text { Men-to-women } \\
\text { ratio }\end{array}$ \\
& $0.0 \%$ & $0.4 \%$ & - \\
$<35 y$ & $5.6 \%$ & $4.2 \%$ & 1.33 \\
$35-45 y$ & $6.3 \%$ & $7.0 \%$ & 0.90 \\
$45-55 y$ & $11.3 \%$ & $11.8 \%$ & 0.95 \\
$55-65 y$ & $15.8 \%$ & $10.5 \%$ & 1.50 \\
$65-75 y$ & $10.8 \%$ & $16.7 \%$ & 0.64 \\
$>75 y$ & $5.8 \%$ & $5.3 \%$ & 1.09 \\
Overall & & & \\
\hline
\end{tabular}

model; Table 3). Specifically, multiple logistic regression analysis showed that 1-year difference in baseline age was associated with $4 \%$ higher risk of developing diabetes during a 5 -year period, $10 \mathrm{~cm}$ difference in baseline measurements of waist was associated with $22 \%$ higher risk of diabetes, $10 \mathrm{mg} / \mathrm{dl}$ increase in baseline blood glucose levels were associated with $62 \%$ higher risk of diabetes, and presence of family history of diabetes increased the risk of developing the disease 2.65-fold (Table 3). At this point it should be clarified that body mass index was also a significant predictor of diabetes (OR per $1 \mathrm{~kg} / \mathrm{m}^{2}=1.10,95 \%$ CI $1.03-1.17$ ); however, since body mass index was strongly correlated with waist ( $r=0.74, p<0.0001)$ and in order to avoid co-linearity, we decided to keep waist circumference in the model since it showed the best explanatory ability among all anthropometric measurements (ie, better goodness-of-fit of the estimated models that also included age, sex, physical activity status dietary habits; -2loglikelihood for body mass index, and waist circumference were: 455.9 and 406.8, respectively). Furthermore, abnormal waist (ie, $>102 \mathrm{~cm}$ for men and $88 \mathrm{~cm}$ for women) was associated with 1.52-times higher risk of diabetes (95\% CI 0.88-2.78). No difference in the predictive ability of anthropometric measurements was observed between sexes ( $p$ for interaction with sex $>0.7$ ). Physical activity was inversely associated with the development of diabetes, since participants that reported engagement in any activity were 0.62-times less likely to develop diabetes. Furthermore, stratified analysis showed that moderate physical activity reduced the odds of diabetes by $59 \%(p=0.04)$, while vigorous physical activity did not show any significance $(\mathrm{OR}=1.29,95 \%$ CI $0.58-2.88)$. All the aforementioned relationships were independent from sex, education status, blood lipids and C-reactive protein levels, presence and management of hypertension and hypercholesterolemia, dietary habits, and smoking status, as measured at baseline examination.

Furthermore, the 5-year incidence of diabetes among people with the metabolic syndrome was $12.9 \%$, while the incidence among people without the syndrome was $3.9 \%$ $(\mathrm{p}<0.001)$. Multi-adjusted data analysis showed that presence of the metabolic syndrome at baseline evaluation 2.95-fold the risk of diabetes (95\% CI 1.89-4.61). Moreover, the model that contained the metabolic syndrome had better classification ability than the model that contained the components of the syndrome (ie, correct classification rate: $94.5 \%$ vs $92.3 \%$ ).

\section{Discussion}

In this work we presented the results regarding diabetes incidence from the ATTICA epidemiological study that has been contacted in a Greek population. The findings show that the incidence of diagnosed type 2 diabetes in our adult sample increased between 2001 and 2006 by 5.5\%. Particularly, the age-adjusted incidence of diabetes was $5.8 \%$ in men and $5.2 \%$ in women, which means that the Greek diabetes population increased by 330,000 people during the five preceding years. This increase in diabetes was mainly a result of aging, abnormal anthropometric measurements, and especially high waist circumference, glucose levels, family history of diabetes, and physical inactivity. However, it should be underlined that these figures may reflect a true increase in the incidence of diabetes, or an increase in the screening for diabetes, or a combination of both. However, rates of diabetes most likely reflect an increase in the true prevalence (ie, diagnosed plus undiagnosed), because changes on the trends of undiagnosed diabetes over the relatively short period appear unlikely to happen (Eliasson et al 2002).

Previous data from Greece suggests that the prevalence of the adult population with known diabetes increased from 250,000 in 1974 (Katsilambros et al 1993) to 800,000 in 2000s (Gikas et al 2004; Panagiotakos et al 2005d), representing a 3-fold increase of the Greek diabetic population over the last 3 decades. We observed a considerable increase in the prevalence of diabetes of about $1 \%$ annually. These findings are in accordance with results of other epidemiologic studies in North America and the Mediterranean region, which suggests that, apart from the increase in the prevalence of diabetes, an increased incidence of diabetes also occurred during the last 30 years worldwide (Geiss et al 2006; Fox et al 2006; Evans et al 2007). For example, the prevalence of diabetes in Greece is close to the prevalence in USA (ie, 9.6\%) (Geiss et al 2006), in Spain (ie, 11\%) (Velverde 
Table 2 Baseline characteristics of the ATTICA study's participants according to the 5 -year incidence of type 2 diabetes mellitus

Status at 5-year follow-up

\begin{tabular}{|c|c|c|c|}
\hline Baseline factors: & $\begin{array}{l}\text { Nondiabetic } \\
(n=1706)\end{array}$ & $\begin{array}{l}\text { Diabetic } \\
(n=100)\end{array}$ & $\mathbf{P}$ \\
\hline Age, $y$ & $43 \pm 11$ & $53 \pm 13$ & 0.001 \\
\hline Male sex, \% & $49 \%$ & $51 \%$ & 0.64 \\
\hline Years of school & $13 \pm 3$ & $10 \pm 4$ & 0.001 \\
\hline Smokers, \% & $42 \%$ & $31 \%$ & 0.03 \\
\hline Physically inactive subjects, $\%$ & $58 \%$ & $63 \%$ & 0.33 \\
\hline Mediterranean diet score $(0-55)$ & $26 \pm 5$ & $23 \pm 6$ & 0.001 \\
\hline Ethanol intake, g/day & $8.7 \pm 12$ & $11.3 \pm 18$ & 0.38 \\
\hline Hypertensive subjects, \% & $28 \%$ & $45 \%$ & 0.001 \\
\hline Systolic blood pressure, $\mathrm{mmHg}$ & $12 \mid \pm 18$ & $128 \pm 17$ & 0.001 \\
\hline Diastolic blood pressure, $\mathrm{mmHg}$ & $79 \pm 8$ & $84 \pm 9$ & 0.02 \\
\hline Hypercholesterolemic subjects, \% & $39 \%$ & $55 \%$ & 0.001 \\
\hline Total cholesterol, mg/dl & $|93 \pm 4|$ & $208 \pm 42$ & $<0.001$ \\
\hline HDL-cholesterol, mg/dl & $49 \pm 13$ & $45 \pm 14$ & 0.04 \\
\hline LDL-cholesterol, mg/dl & $122 \pm 37$ & $135 \pm 40$ & 0.002 \\
\hline Triglycerides, mg/dl & $111 \pm 67$ & $156 \pm 70$ & 0.001 \\
\hline Fasting glucose, mg/dl & $89 \pm 19$ & $97 \pm 18$ & 0.001 \\
\hline hs C-reactive protein, mg/l & $1.8 \pm 2.3$ & $2.5 \pm 2.7$ & 0.01 \\
\hline Obese subjects, \% & $16 \%$ & $39 \%$ & 0.001 \\
\hline Body mass index, $\mathrm{kg} / \mathrm{m}^{2}$ & $26 \pm 5$ & $29 \pm 4$ & 0.001 \\
\hline \multicolumn{4}{|l|}{ Waist, $\mathrm{cm}$} \\
\hline Men & $96 \pm 11$ & $104 \pm 12$ & 0.001 \\
\hline Women & $82 \pm 10$ & $90 \pm 11$ & 0.001 \\
\hline
\end{tabular}

Notes: Data are expressed as mean \pm SD or relative frequencies. Abbreviations: HDL, high-density lipoprotein; LDL, low-density lipoprotein.

et al 2006), and in Cyprus (ie, 10.3\%) (Loizou et al 2006). Moreover, the observed trend is in accordance to the estimated projection of diabetes prevalence reported by Wild and colleagues (2004) (ie, a 57\% increase from 2000 to 2030). We have also observed that the adjusted incidence of diabetes increases by $4 \%$ per 1 year difference in age or by $48 \%$ per decade of life. It could be speculated that the higher increase of diabetes in elderly may reflect the simultaneously higher increase in obesity rates and other co-morbidities related to diabetes (Dinsmoor 2006). Furthermore, we observed that the diabetes incidence was much higher among persons with low education (Table 2). This finding is in accord with other reports (Kanjilal et al 2006), and may reflect the higher prevalence of cardiovascular risk factors, especially obesity, among people with low socioeconomic status (Panagiotakos et al 2005b). This could also be an explanation why the education status did not show any significance in the multiadjusted model where several other factors were also taken into account (Table 3). Nevertheless, prevention strategies against diabetes epidemic need to specifically target persons of lower education.

Several studies have shown that diabetes prevalence is strongly associated with the presence of obesity (WHO 1997; Kahn et al 2006; Yoon et al 2006) and the metabolic syndrome (WHO 1997; Ford et al 2002; Panagiotakos et al 2005c). In a recent review paper, Kahn and colleagues (2006) suggested that among obese individuals adipose tissue releases high amounts of nonesterified fatty acids, glycerol, hormones, proinflammatory cytokines, and other factors that are involved in the development of insulin resistance and diabetes. Our study revealed that the incidence of diabetes was strongly associated with all the investigated anthropometric indices and waist circumference showed the most significant association with diabetes rates. Abdominal obesity has been associated with increased risk of cardiovascular disease and diabetes, mainly, through the development of many metabolic disorders 
Table 3 Results from multiple logistic regression analysis that evaluated sociodemographic, lifestyle, biological, clinical baseline characteristics in relation to 5 -year incidence of type 2 diabetes in the ATTICA study participants

\begin{tabular}{|c|c|c|}
\hline & Odds ratio & $\begin{array}{l}\text { 95\% Confidence } \\
\text { interval }\end{array}$ \\
\hline \multicolumn{3}{|l|}{ Initial model } \\
\hline Age (per I year) & 1.04 & $1.02-1.07$ \\
\hline Male vs. females & 1.24 & $0.35-4.37$ \\
\hline Years of school (per I year) & 0.94 & $0.8 I-I .10$ \\
\hline Smokers vs. nonsmokers & 0.84 & $0.28-2.25$ \\
\hline \multicolumn{3}{|l|}{ (per I unit) } \\
\hline Ethanol intake (per I g/d) & 1.002 & $0.97-1.03$ \\
\hline Physically active vs. inactive & 0.40 & $0.12-1.31$ \\
\hline Waist circumference (per I cm) & 1.05 & $1.01-1.09$ \\
\hline \multicolumn{3}{|l|}{$($ per $I \mathrm{mg} / \mathrm{dl})$} \\
\hline Hypercholesterolemia $(y / n)$ & 0.59 & $0.17-2.05$ \\
\hline Hypertension $(y / n)$ & 0.42 & $0.12-1.45$ \\
\hline Family history of diabetes $(y / n)$ & 3.93 & $1.30-11.9$ \\
\hline hs C-reactive protein (per I mg/l) & 1.06 & $1.87-1.28$ \\
\hline \multicolumn{3}{|l|}{ Final model } \\
\hline Age (per I year) & 1.04 & $1.02-1.07$ \\
\hline Waist (per I cm) & 1.02 & $1.01-1.03$ \\
\hline Physically active vs. inactive & 0.62 & $0.35-1.02$ \\
\hline Fasting blood glucose (per I mg/dl) & 1.05 & $1.03-1.07$ \\
\hline Family history of diabetes $(y / n)$ & 2.65 & $1.58-4.53$ \\
\hline
\end{tabular}

Notes: $-2 \log$ (likelihood) for the initial model $=470.6$ and for the final model $=475.4$ ( $p$ for the differences in -2loglikelihoods $=0.9$ ).

characterized by insulin resistance, including hypertension, as well as alterations in homeostasis, fibrinolysis, and inflammation (WHO 1997; Panagiotakos et al 2005c).

The metabolic syndrome is also a condition, which is known that promotes atherosclerosis and increases the risk of cardiovascular disease and diabetes (Ford et al 2002; Haffner 2006; Grundy 2006). The two major underlying risk factors for the syndrome are obesity and insulin resistance. Both factors are known that promote the development of type 2 diabetes. In this work, we revealed that presence of metabolic syndrome almost 3-fold the risk for diabetes in both sexes and independent from other risk factors. Moreover, we observed that the metabolic syndrome was better predictor of diabetes than the individual components of the syndrome, which suggests that the clustering of conditions that define the syndrome is of major importance.

From the early 1980s, several observational studies suggest that mortality or morbidity caused by atherosclerotic disease was inversely related to the individuals' physical activity status (Kannel 1976; Leon et al 1987; Pekkanen 1987). Even a single session of moderate to long-duration exercise can reduce blood pressure, glucose, and triglycerides levels, and can increase HDL-cholesterol levels. As a consequence, physical activity may moderate the levels of the clinical and biochemical components of the metabolic syndrome and, therefore reduce its prevalence at population level. Through this mechanism, physical activity may also reduce the risk for developing diabetes. We observed that presence of physical activity was associated with $38 \%$ lower risk of diabetes, irrespective of age, sex, body mass index, blood glucose levels, and other potential confounders (Table 3). We have also found that the effect of moderate exercise was more significant than the influence of vigorous activities in the prevention of diabetes. The NCEP ATP III suggested therapeutic lifestyle changes (NCEP 2001) in order to reduce the prevalence of the metabolic syndrome and diabetes, underlying the importance of low-saturated diet consumption and physical exercise. Thus, public health actions that promote physical activity and healthy eating should implemented in social programs starting from school years.

Finally, it is known that family history of diabetes is strongly associated with the development of the disease, implying a genetic predisposition (Harrison et al 2003). We observed that people who had a first degree relative with type 2 diabetes experience 2.65 -times higher risk of diabetes, compared with those that did not have history of the disease in their family. However, diabetes is a complex condition that involves a combination of genetic and environmental factors; thus, DNA testing for susceptibility genes is not yet warranted. Despite these considerations, Harisson and colleagues (2003) suggested that since family history of diabetes reflects genetic susceptibility in addition to other factors, it could be a useful public health tool for disease prevention.

\section{Limitations}

This work has some limitations. At first the baseline evaluation was performed once, and may be prone to measurement error. Moreover, we have excluded those with coronary heart disease at baseline, which are usually on beta-blockers or/and diuretics. Thus, the incidence of type 2 diabetes may have been significantly influenced in our sample. Additionally, telephone call for the ascertainment of diabetes is valid only when accurate medical records exist; thus, individuals that may develop diabetes during the 5-year period, but they did not know it, were misclassified in the re-examination (ie, underestimation of the actual incidence rates). However, $97 \%$ of the participants that were found in the follow-up provided accurate medical records, and reported that they have checked their blood glucose levels at least once during the preceding period. Furthermore, our methodology is similar to those of other cross-sectional surveys 
and follow-up epidemiological studies in Europe and the US, and therefore our results are comparable. Another limitation could be the fact that the relative risks were estimated by the odds ratios through multiple logistic regression analysis. This may overestimate the actual risk. Finally, misreporting of alcohol consumption at the baseline evaluation, due to social class, can be a potential confounder in the evaluation of alcohol intake on the development of diabetes.

\section{Conclusion}

Our findings show that the prevalence of diabetes has risen in the Greek population during the past 5 years. Given the aging population, these data suggest that the growing burden of diabetes will be extremely dynamic over the next years. To reverse this trend, a sustained and effective public health response is needed to focus on weight loss and increase physical activity by promoting lifestyle changes.

\section{Acknowledgments}

The ATTICA study was supported by research grants from the Hellenic Cardiological Society (HCS2002). The authors would like to thank the ATTICA study investigators: M. Toutouza (biochemical analyses, head), A. Zeimbekis (physical examination), N. Papaioannou (physical examination), E. Tsetsekou (physical examination), C. Massoura (physical examination), S. Vellas (physical examination), L. Papadimitriou (physical examination), C. Vassiliadou (genetic evaluation), E. Kambaxis (nutritional evaluation), K. Paliou (nutritional evaluation), K. Tselika (biochemical analyses), S. Poulopoulou (biochemical analyses), and M. Toutouza (database management).

\section{References}

[CDC] Centers for Disease Control and Prevention. 2005. National diabetes fact sheet: general information and national estimates on diabetes in the United States, 2005. Atlanta, GA: US Department of Health and Human Services, CDC.

Dinsmoor RS. 2006. Healthy aging with diabetes. Diabetes Self Manag, 23:20-2.

Eliasson M, Lindahl B, Lundberg V, et al. 2002. No increase in the prevalence of known diabetes between 1986 and 1999 in subjects 25-64 years of age in northern Sweden. Diabet Med, 19:874-80.

Evans JM, Barnett KN, Ogston SA, et al. 2007. Increasing prevalence of type 2 diabetes in a Scottish population: effect of increasing incidence or decreasing mortality? Diabetologia, 50:729-32.

Ford ES, Giles WH, Dietz WH. 2002. Prevalence of the metabolic syndrome among US adults: findings from the third National Health and Nutrition Examination Survey. JAMA, 287:356-9.

Fox CS, Pencina MJ, Meigs JB, et al. 2006. Trends in the incidence of type 2 diabetes mellitus from the 1970s to the 1990s: the Framingham Heart Study. Circulation, 113:2914-18.

Geiss LS, Pan L, Cadwell B, et al. 2006. Changes in incidence of diabetes in U.S. adults, 1997-2003. Am J Prev Med, 30:371-7.
Gikas A, Sotiropoulos A, Panagiotakos D, et al. 2004. Prevalence, and associated risk factors, of self-reported diabetes mellitus in a sample of urban population in Greece: MEDICAL Exit Poll Research in Salamis (MEDICAL EXPRESS 2002). BMC Public Health, 4:2.

Grundy SM. 2006. Metabolic syndrome: connecting and reconciling cardiovascular and diabetes worlds. J Am Coll Cardiol, 47:1093-100.

Haffner SM. 2006. The metabolic syndrome: inflammation, diabetes mellitus, and cardiovascular disease. Am J Cardiol, 97:3A-11A.

Harrison TA, Hindorff LA, Kim H, et al. 2003. Family history of diabetes as a potential public health tool. Am J Prev Med, 24:152-9.

IPAQ. 2006. International Physical Activity Questionnaire [online]. Accessed on February 28, 2006. URL: http://www.ipaq.ki.se/ipaq.htm.

Kahn SE, Hull RL, Utzschneider KM. 2006. Mechanisms linking obesity to insulin resistance and type 2 diabetes. Nature, 444:840-6.

Kanjilal S, Gregg EW, Cheng YJ, et al. 2006. Socioeconomic status and trends in disparities in 4 major risk factors for cardiovascular disease among US adults, 1971-2002. Arch Intern Med, 166:2348-55.

Kannel WB, McGee DL, Gordon T. 1976. A general cardiovascular risk profile: The Framingham Study. Am J Cardiol, 38:46-51.

Katsilambros N, Aliferis K, Darviri CH, et al. 1993. Evidence for an increase in the prevalence of known diabetes in a sample of an urban population in Greece. Diabet Med, 10:87-90.

Katsouyanni K, Rimm EB, Gnardellis C, et al. 1997. Reproducibility and relative validity of an extensive semi-quantitative food frequency questionnaire using dietary records and biochemical markers among Greek schoolteachers. Int J Epidemiol, 26:S118-27.

King H, Aubert RE, Herman WH. 1998. Global burden of diabetes, 1995-2025. Diabetes Care, 21:1414-31.

Leon AS, Connett J, Jacobs DR, et al. 1987. Leisure-time physical activity levels and risk of coronary heart disease and death: the Multiple Risk Factor Intervention Trial. JAMA, 258:2388-95.

Loizou T, Pouloukas S, Tountas C, et al. 2006. An epidemiologic study on the prevalence of diabetes, glucose intolerance, and metabolic syndrome in the adult population of the Republic of Cyprus. Diabetes Care, 29:1714-15.

Mayor S. 2006. Diabetes affects nearly 6\% of the world's adults. BMJ, 333:1191.

[NCEP] National Cholesterol Education Program. 2001. Executive Summary of the Third Report of the National Cholesterol Education Program (NCEP) Expert Panel on Detection, Evaluation, and Treatment of High Blood Cholesterol in Adults (Adult Treatment Panel III). JAMA, 285:2486-97.

Panagiotakos DB, Pitsavos C, Chrysohoou C, et al. 2005a. The epidemiology of type 2 diabetes mellitus in Greek adults: the ATTICA study. Diabet Med, 22:1581-8.

Panagiotakos DB, Pitsavos C, Manios Y, et al. 2005b. Socio-economic status in relation to risk factors associated with cardiovascular disease, in healthy individuals from the ATTICA study. Eur J Cardiovasc Prev Rehabil, 12:68-74.

Panagiotakos DB, Pitsavos C, Stefanadis C. 2006. Dietary patterns: a Mediterranean diet score and its relation to clinical and biological markers of cardiovascular disease risk. Nutr Metab Cardiovasc Dis, 16:559-68.

Panagiotakos DB, Pitsavos C, Yannakoulia M, et al. 2005c. The implication of obesity and central fat on markers of chronic inflammation: The ATTICA study. Atherosclerosis, 183:308-15.

Panagiotakos DB, Tzima N, Pitsavos C, et al. 2005d. The relationship between dietary habits, blood glucose and insulin levels among people without cardiovascular disease and type 2 diabetes; The ATTICA study. Rev Diabet Stud, 2:208-15.

Patja K, Jousilahti P, Hu G, et al. 2005. Effects of smoking, obesity and physical activity on the risk of type 2 diabetes in middle-aged Finnish men and women. J Intern Med, 258:356-62.

Pekkanen J, Marti B, Nissinen A, et al. 1987. Reduction of premature mortality by high physical activity: a 20-year follow-up of middle-aged Finnish men. Lancet, 1987; 255:1473-7. 
Pitsavos C, Panagiotakos DB, Chrysohoou C, et al. 2003. Epidemiology of cardiovascular risk factors in Greece: aims, design and baseline characteristics of the ATTICA study. BMC Public Health, 3:32.

Tuomilehto J, Wareham N. 2006. Glucose lowering and diabetes prevention: are they the same? Lancet, 368:1218-19.

Valverde JC, Tormo MJ, Navarro C, et al. 2006. Prevalence of diabetes in Murcia (Spain): a Mediterranean area characterised by obesity. Diabetes Res Clin Pract, 71:202-9.

van Dam RM, Rimm EB, Willett WC, et al. 2002. Dietary patterns and risk for type 2 diabetes mellitus in U.S. men. Ann Intern Med, 136:201-9.
Wild S, Roglic G, Green A, et al. 2004. Global prevalence of diabetes: estimates for the year 2000 and projections for 2030. Diabetes Care, 27:1047-53.

[WHO] World Health Organization. 1997. Obesity: preventing and managing the global epidemic. Report of a WHO Consultation presented at: the World Health Organization; June 3-5, 1997; Geneva, Switzerland, WHO. Publication WHO/NUT/NCD/98.1.

[WHO] World Health Organization. 2002. Diabetes mellitus Fact Sheet No 138, revised April 2002. Geneva, Switzerland, WHO.

Yoon KH, Lee JH, Kim JW, et al. 2006. Epidemic obesity and type 2 diabetes in Asia. Lancet, 368:1681-8. 